\title{
Model of the meniscus of an ionic-liquid ion source
}

\author{
F. J. Higuera \\ ETS Ingenieros Aeronáuticos, UPM, Plaza Cardenal Cisneros 3, 28040 Madrid, Spain
}

\begin{abstract}
A simple model of the transfer of charge and ion evaporation in the meniscus of an ionic-liquid ion source working in the purely ionic regime is proposed on the basis of order-of-magnitude estimates which show that, in this regime, (i) the flow in the meniscus is dominated by the viscosity of the liquid and is affected very little by the mass flux accompanying ion evaporation, and (ii) the effect of the space charge around the evaporating surface is negligible and the evaporation current is controlled by the finite electrical conductivity of the liquid. The model predicts that a stationary meniscus of a very polar liquid undergoing ion evaporation is nearly hydrostatic and can exist only below a certain value of the applied electric field, at which the meniscus attains its maximum elongation but stays smooth. The electric current vs applied electric field characteristic displays a frozen regime of negligible ion evaporation at low fields and a conduction-controlled regime at higher fields, with a sharp transition between the two regimes owing to the high sensitivity of the ion evaporation rate to the electric field. A simplified treatment of the flow in the capillary or liquid layer through which liquid is delivered to the meniscus shows that the size of the meniscus decreases and the maximum attainable current increases when the feeding pressure is decreased, and that appropriate combinations of feeding pressure and pressure drop may lead to high maximum currents.
\end{abstract}

\section{INTRODUCTION}

Liquid metals and molten or dissolved salts may undergo ion evaporation from their surfaces in the presence of a high electric field. This phenomenon is at the base of the generation of ion beams from ion sources of these materials. Liquid metal ion sources (LMISs) are the most extensively used and studied of these sources, with applications ranging from microfabrication and analytical instrumentation to electric propulsion. In the most common configuration the liquid metal covers the roughened surface of a thin metallic needle held in a vacuum, and flows by capillary action toward the electrochemically sharpened tip of the needle, where a small meniscus is formed. A high voltage is applied between the needle and a neighboring extractor electrode shaped as a plane perpendicular to the needle with an orifice in front of it. The electric field is intensified by the needle, leading to electric stresses on the surface of the liquid that strain the meniscus and further intensify the field. Ion evaporation occurs in a region of a few nanometers around the tip of the meniscus, where the electric field is of the order of 10 $\mathrm{V} / \mathrm{nm}$. The space charge due to the evaporated ions plays an important role in this region, limiting the electric field on the surface and the evaporation rate, and causing the meniscus to develop a characteristic protrusion.

Ion sources of nonmetallic liquids, with electrical conductivities much smaller than those of metals, are a more recent development that originated in the electrospray technique.

In the

simplest configuration, the liquid to be electrosprayed forms a meniscus at the end of a capillary through which it is fed. The meniscus is electrically stressed much as described above for a LMIS until it evolves into a conical shape (a Taylor cone) whose tip ejects a narrow jet that eventually breaks into a spray of monodisperse drops. Owing to the moderate conductivity of the liquid, the surface of the jet is not an equipotential surface, and the electric shear due to the action of the component of the electric field tangent to the surface on the electric charge that the field itself accumulates at the surface strains the jet and generally confers stability on it. The diameter of the jet (and the size of the drops) decreases, and the maximum electric field on the surface of the liquid increases, when the flow rate of the liquid fed into the meniscus decreases or the electrical conductivity of the liquid increases. The diameter of the jet can be controlled and reduced down to about $10 \mathrm{~nm}$ by acting on these parameters

which makes the electrospray useful as a source of drops for a variety of applications, especially in mass spectrometry as a nondestructive way to obtain intact macromolecules and also as a colloid thruster for electric propulsion with a specific impulse that increases as the mass of the charged drops decreases.

Many electrolytic solutions and ionic liquids (which are molten salts at ambient temperature) begin to undergo ion evaporation around the tip of the electrospray meniscus when the maximum electric field reaches values in the range of $1 \mathrm{~V} / \mathrm{nm}$. Positive or negative ions can be obtained by this means with a wide variety of chemical compositions and mass-to-charge ratios, which are key parameters determining the properties and breadth of applications of the different ion beams. For example, a beam of ions with mass-to-charge ratio well above the 200 daltons attainable with LMISs would decrease the space charge limitations that oppose sharp focusing, and thus would allow intense fluxes of ions for localized erosion or deposition; ions of increased mass are of interest for electric propulsion, to reduce the energy consumed per unit thrust; and ions of suitable chemical composition could allow a combination of physical erosion with chemical attack in applications such as etching.

In most of the work carried out with the capillary type of source, ions coexist with charged drops, and the fraction of ions increases when the conductivity of the liquid is increased or its flow rate is decreased Exceptions 
where a purely ionic regime (ions without drops) has been reported are the work of Perel et al. with concentrated sulfuric acid solutions and the work of Romero-Sanz et al. with the ionic liquid $l$-ethyl-3-methyl imidazolium

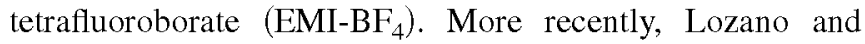
Martínez-Sánchez adapted the externally wetted needle configuration of LMISs to ionic-liquid ion sources (ILISs) and showed that the purely ionic regime can be achieved with many other ionic liquids in these new sources, with narrow beam energy distributions and low energy deficits comparable to those of LMISs;

Comparisons of capillary and externally wetted needle configurations, and details of the fabrication of emitters for the latter type of source, are given by Lozano and Martínez-Sánchez

The continuous extraction of ions of a given polarity (anions or cations) from an ionic liquid causes accumulation of their counterions, which are neutralized at the needle or capillary electrode and may either react with this electrode or stay in solution; for a recent review of the electrochemistry of electrosprays. These processes drastically limit the lifetime of the source by degrading the electrode or the liquid. Lozano and Martínez-Sánchez showed that the electrochemical reactions of the nonextracted species can be avoided through alternation of the applied voltage with a frequency dictated by the charging time of the double layer generated at the liquid-metal interface. The minimum required frequency depends on the electrochemical window of the liquid [20], but typically is in the range of $1 \mathrm{~Hz}$. The dynamic response of the meniscus, which has to reform two times per cycle, is much faster [22]. Ion beams generated by this means are bipolar, containing positive and negative ions, which would not be possible with LMISs (limited to positive ions) and is very useful in applications like electric propulsion, where nonextracted counterions represent a loss of propulsive mass, and others where accumulation of charge is not admissible.

In this paper, an order-of-magnitude analysis is used to estimate the effects of the space charge and the mass flux accompanying ion evaporation on the dynamics of the meniscus and the distribution of electric current in the purely ionic regime of an ILIS. Both effects are found to be small. A simplified model of the flow in the meniscus is proposed in which these effects and the effect of the inertia of the liquid are omitted. In addition, the capillary or needle configuration of a real source is replaced by a parallel plate configuration, in order to simplify the numerical treatment of the mathematical problem, and the details of the flow feeding the meniscus are lumped into an effective pressure drop coefficient. Because of these and other simplifications discussed below, the model cannot describe a real ILIS, but it still provides qualitative information on key issues such as the role of the finite electrical conductivity of the liquid in controlling the emitted current, the size and shape of the ionemitting surface, the existence of a starting voltage, and the dependence of the current-voltage characteristic and the maximum attainable current on the feeding pressure and the hydraulic resistance of the effective feeding line.

\section{MODEL SETUP}

\section{A. Ion evaporation}

Evaporation of ions from the charged surface of a nonmetallic liquid in which the ions are dissolved is a process first described by Iribarne and Thomson which presents itself in a variety of applications. A certain amount of energy is required to bring a (possibly solvated) ion from the liquid to a large distance away from its surface. This energy is used to form a new surface and to overcome the electrostatic attraction of the image charge left behind by the evaporating ion. In the absence of an external electric field, the required energy is large compared with the thermal energy of the ions in the liquid, so that the rate of ion evaporation is very small. An external field decreases the electrostatic barrier for ions of the appropriate polarity, and may increase their evaporation rate to appreciable values.

The rate of ion evaporation (electric charge crossing the surface per unit area and time) is

$$
j_{e}=\frac{k T}{h} \sigma \exp \left(-\frac{\mathcal{E}}{k T}\right),
$$

where $h$ and $k$ are the Planck and Boltzmann constants, $T$ is the temperature of the liquid, $\sigma$ is the density of free charge at its surface, and $\mathcal{E}$ is the activation energy of the evaporation process. This activation energy has been determined using a variety of approximations For a very polar liquid with a surface of small curvature, the activation energy can be written in the form

$$
\mathcal{E}=\Delta G-G\left(E_{n}\right) \quad \text { with } G\left(E_{n}\right)=\left(\frac{e^{3} E_{n}}{4 \pi \epsilon_{0}}\right)^{1 / 2},
$$

where $\Delta G$ is the solvation energy of the ions, $E_{n}$ is the external field normal to the surface, and $e$ and $\epsilon_{0}$ are the elementary charge and the permittivity of vacuum.

The values of the surface field at which ion evaporation occurs are typically large compared to $E_{D}=\left(\epsilon n_{0} k T / \epsilon_{0}\right)^{1 / 2}$, which is the order of the maximum surface field for which ions of both polarities still coexist in the Debye layer at the liquid surface with number densities of the order of their value $n_{0}$ in the bulk of the liquid Here $\epsilon$ is the dielectric constant of the liquid. When $E_{n}$ is large compared to $E_{D}$, the non-neutral layer responsible for the surface charge includes a sublayer of characteristic thickness $\epsilon k T / e E_{n}$ where positive ions accumulate with a density of order $n_{0}\left(E_{n} / E_{D}\right)^{2}$.

\section{B. Orders of magnitude}

Values of the solvation energy of the ionic liquids and solutions of interest for electric propulsion have not been accurately determined but are expected to be in the range $\Delta G \approx 1.4-2 \mathrm{eV}$ or above leading to $\Delta G / k T=54-78$ or higher at room temperature. These high values make the exponent in (1) very large and render ion evaporation negligible in the absence of an electric field.

To estimate the order of the electric field needed at the surface of the liquid for ion evaporation to play a role in the transfer of electric charge, consider first the electrostatic con- 
dition $\epsilon_{0}\left(E_{n}-\epsilon E_{n}^{l}\right)=\sigma$ at the surface of the liquid

which links the density of free surface charge with the components of the electric field normal to the surface outside and inside the liquid ( $E_{n}$ and $E_{n}^{l}$ respectively). The latter field is negligible in equilibrium in the absence of evaporation, when the surface charge attains its maximum value $\epsilon_{0} E_{n}$ and screens the liquid from the external field. On the other hand, the maximum value of the normal component of the internal field is $E_{n}^{l}=E_{n} / \epsilon$, which is attained in the absence of free surface charge. The internal field $\left(\boldsymbol{E}^{l}\right.$ in what follows) induces a conduction current in the liquid which will be assumed to obey Ohm's law with a constant conductivity $K$, though this assumption is not without limitations in the conditions of an ion source; see Ref. [4] for criticism and a more accurate modeling of the electrical conduction, and also the comments at the end of this section. Using Ohm's law as an approximation, the electric current density is $\boldsymbol{j}=K \boldsymbol{E}^{l}$, whose component normal to the surface is $\dot{j}_{c}$ $=K E_{n}^{l}$. The condition for the ion evaporation current to play a role is $j_{e} \sim j_{c_{\max }}$, with $j_{e}$ given by (1) and $j_{c_{\max }}=K E_{n} / \epsilon$. When $\sigma \sim \epsilon_{0} E_{n}$ and $E_{n}^{l} \sim E_{n} / \epsilon$, this order-of-magnitude condition amounts to

$$
\frac{k T}{h} \epsilon_{0} E_{n} \exp \left(-\frac{\Delta G-G\left(E_{n}\right)}{k T}\right) \sim K \frac{E_{n}}{\epsilon}
$$

or

$$
G\left(E_{n}\right) \approx \widetilde{\Delta G} \text { with } \widetilde{\Delta G}=\Delta G+k T \ln \frac{K / \epsilon_{0} \epsilon}{k T / h}
$$

At room temperature $\widetilde{\Delta G}$ is in the range $1.2-1.8 \mathrm{eV}$ for $K$ $=1 \mathrm{~S} / \mathrm{m}$ and $\epsilon=64.92$, which are values typical of solutions of some ionic liquids in propylene carbonate. This leads to $E_{n} \approx 1-2 \mathrm{~V} / \mathrm{nm}$.

In an ILIS, high fields of this order are attained around the tip of the meniscus, whose shape is determined by the balance of surface tension and electric stresses. The normal electric stress acting on an equipotential liquid surface is $\frac{1}{2} \epsilon_{0} E_{n}^{2}$ [see (9a) and (9b) below for a more general expression]. The normal stress due to the surface tension is $\gamma / r_{v}$, where $\gamma$ is the surface tension of the liquid and $1 / r_{v}$ is twice the mean curvature of its surface. The balance of these stresses gives $r_{v} \sim 2 \gamma / \epsilon_{0} E_{n}^{2}$ for the characteristic size of the region around the tip of the meniscus where ion evaporation occurs, which is of the order of $10 \mathrm{~nm}$ for a liquid with the surface tension of propylene carbonate $(\gamma=4.19$ $\left.\times 10^{-2} \mathrm{~N} / \mathrm{m}\right)$ and the value of $E_{n}$ estimated above.

The characteristic evaporation current can now be estimated as $I_{e} \sim 2 \pi j_{e} r_{v}^{2}=8 \pi \gamma^{2} K /\left(\epsilon_{0}^{2} E_{n}^{3}\right)$, where $j_{e} \sim K E_{n} / \epsilon$ according to the balance leading to (3) and the estimate of $r_{v}$ above has been used. This current is of the order of $10 \mathrm{nA}$, which is small compared with the currents measured for the most promising solutions of ionic liquids in the range of hundreds of nanoamperes. As possible causes of the discrepancy, Fernández de la Mora points out that the solvation energy could be smaller than assumed above, which would lead to a lower $E_{n}$ and a higher $I_{e}$, and that the electrical conductivity could be larger than assumed owing to the increase of the liquid temperature by Joule dissipation.
The first possibility is consistent with some results of Ref.

The order of the temperature increment due to Joule dissipation can be estimated from the thermal energy balance $\lambda \nabla^{2} T+\boldsymbol{j} \cdot \boldsymbol{E}^{l}=0$, where $\lambda$ is the thermal conductivity of the liquid and $\boldsymbol{j} \cdot \boldsymbol{E}^{l} \sim\left(I_{e} / r_{v}^{2}\right)\left(E_{n} / \epsilon\right)$. This gives $\Delta T \sim I_{e} E_{n} / \lambda \epsilon$, which is of the order of a few kelvins for a liquid with the thermal conductivity of propylene carbonate $(\lambda$ $=0.16 \mathrm{~W} / \mathrm{m} \mathrm{K}$ ) and a current of $10 \mathrm{nA}$, but increases proportionally to the current and may rise to tens or even hundreds of kelvins for the highest currents measured experimentally, thereby having a noticeable effect on the electrical conductivity of the liquid in such cases.

The mass flux accompanying ion evaporation is $(\mathrm{m} / q) j_{e}$, where $m$ and $q$ are the mass and charge of the evaporating ions. If liquid is continuously supplied to the meniscus to make for the loss of ions by evaporation and the proportional loss of counterions to the electrode, then the velocity induced in the meniscus to transport the evaporating ions to the surface is $v_{v} \sim(m / q) j_{e} /\left(\rho c_{0}\right)$, where $\rho$ is the density of the liquid and $c_{0}$ is the concentration (in mass) of the ions dissociated in the liquid. This velocity is of the order of 3.5 $\times 10^{-2} \mathrm{~m} / \mathrm{s}$ for $\mathrm{m} / q=200$ daltons, $c_{0}=O(1)$, and $\rho=1.2$ $\times 10^{3} \mathrm{~kg} / \mathrm{m}^{3}$, which are typical of not-very-diluted solutions of ionic liquids in propylene carbonate.

On the other hand, the velocity $v_{c}$ of the flow induced in a dynamic meniscus by the surface tension and electric stresses acting on its surface can be estimated from the orderof-magnitude balance of these stresses and viscous stresses: $\mu v_{c} / r_{v} \sim \gamma / r_{v}$, which gives $v_{c} \sim \gamma / \mu$, of the order of $15 \mathrm{~m} / \mathrm{s}$ for propylene carbonate $\left(\mu=2.76 \times 10^{-3} \mathrm{~kg} / \mathrm{m} \mathrm{s}\right)$. It has been assumed here that the effect of the inertia of the liquid is negligible, which is justified by the small value of the Reynolds number $\rho v_{c} r_{v} / \mu$, of the order of 0.03 . The velocity $v_{c}$ is large compared with $v_{v}$, implying that the flow induced in the liquid by ion evaporation does not play an important role in the dynamics of the meniscus.

The exponential factor in (1) is a rapidly increasing function of the electric field. However, the evaporation current cannot increase so rapidly, being limited by the rate at which conduction in the liquid can bring ions to the surface. As a consequence, the density of surface charge $\sigma$ in (1) must decrease very rapidly when the electric field increases, and it becomes very small in the region of the surface where ion evaporation is controlled by conduction.

This controlling mechanism is specific of ionic liquids and other electrolytic solutions whose electrical conductivity is small compared to that of liquid metals. At the much higher conductivities of the liquid metals, ion evaporation is controlled by the space charge surrounding the evaporating surface. Denoting by $n_{\mathrm{sc}}(\boldsymbol{x})$ the number density of evaporated ions in this region, the electric field satisfies $\boldsymbol{\nabla} \cdot \boldsymbol{E}=q n_{\mathrm{sc}} / \boldsymbol{\epsilon}_{0}$. The condition for the field induced by the space charge to be of the order of the external field, in order to effectively reduce the field at the surface, is $E_{n} / r_{v} \sim q n_{\mathrm{sc}} / \epsilon_{0}$, where $E_{n}$ and $r_{v}$ are the characteristic field and size and the evaporation region estimated above. On the other hand, the characteristic velocity of the evaporated ions is $v_{\mathrm{sc}} \sim\left(q E_{n} r_{v} / m\right)^{1 / 2}$, from the energy conservation condition $m v_{\mathrm{sc}}^{2} \sim q E_{n} r_{v}$. The electric current is then of order $q n_{\mathrm{sc}} v_{\mathrm{sc}} r_{v}^{2} \sim \epsilon_{0}\left(E_{n} r_{v}\right)^{3 / 2} /(m / q)^{1 / 2}$. This 
current is of the order of microamperes, which is typical of LMISs but large compared with the current $I_{e}$ estimated above for an ILIS on the basis of liquid conduction control. As a consequence, the density of space charge around the evaporating surface of an ILIS is only of order $I_{e} / q v_{\mathrm{sc}} r_{v}^{2}$, which is too small to appreciably reduce the field at the surface below the field computed in the absence of space charge.

In summary, the order-of-magnitude estimates worked out in this section suggest that the flow in the meniscus of an ILIS is dominated by the viscosity of the liquid and is little affected by the mass flux accompanying ion evaporation, and that the effect of the space charge around the evaporating surface is negligible, with the evaporation current being controlled by the finite electrical conductivity of the liquid.

It may be worth mentioning that a different order-ofmagnitude analysis for LMISs gives results quite different from these. The ion current of a LMIS is limited by the space charge rather than by the finite electrical conductivity of the liquid, and the viscosity of the liquid plays only a secondary role in the dynamics of the meniscus.

The estimates of this section and the analysis that follows in the rest of the paper are for the purely ionic regime. This is not the only possible regime of operation of an ILIS; there is also a mixed regime of drops and ions mentioned in the introduction, in which the meniscus takes a conical shape and ejects a narrow jet. The two regimes, and perhaps a third pulsating regime, might coexist in some regions of the parameter space of the problem, leading to multiplicity, but this possibility will not be analyzed here.

Another complexity of ILISs comes from the presence and evaporation of neutral species. The rate of evaporation of neutral species depends on the nature and temperature of the liquid, and may range from very high values for salts dissolved in relatively volatile solvents such as formamide, to very low values for pure ionic liquids. The estimates above suggest that the flow induced in the meniscus by the transport of solvent is weak and can be neglected together with the flow induced by the evaporation of the ions, except for very dilute solutions, for which the issues of evaporation and/or dripping of the solvent come to the front and require a separate analysis. In any case, the different rates of evaporation of the neutral species and the ions, together with the electrochemical processes occurring at the electrode (if they are not suppressed by voltage alternation), cause slow changes of the composition and properties of the liquid that affect the operation of the source in the long term. These processes also bring in diffusion. Simple estimates

suggest that, if ambipolar diffusion alone were to account for the ion evaporation flux, the concentration of ions would have large spatial variations in the meniscus and the conduction-controlled density of current $K E_{n} / \epsilon$ would be reduced by a factor of order $\epsilon k T / e E_{n} r_{v}$. Ohm's law would not be applicable in these conditions. However, even a modest rate of evaporation of the solvent may cause a highPéclet-number flow that transports salt to the surface more rapidly than diffusion. It suffices for this that the characteristic velocity of such flow be large compared to $D_{a} / r_{v}$, where $D_{a}$ is the coefficient of diffusion of the ions in the liquid. Voltage alternation, on the other hand, may prevent the con-

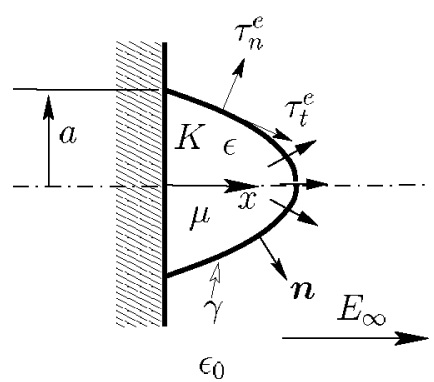

FIG. 1. (Color online) Definition sketch.

tinuous depletion of ions of a given polarity in the vicinity of the liquid surface. The slow changes of the liquid properties with time do not invalidate the analysis that follows; they simply make the parameters of the model depend on the history of the source. Diffusion and spatial variations of ion concentration will be neglected, and the electrical conductivity of the liquid will be taken to be uniform.

\section{Formulation}

Attention is confined here to the flow and the electric current in a meniscus undergoing ion evaporation. On the basis of the preceding estimates, a simple model is proposed that largely isolates the analysis of the meniscus from the complexities of the flow in the capillary or the grooves at the roughened surface of the needle of an ILIS. This latter flow plays an important role in the operation of a source and has been analyzed both for LMISs and for ILISs where its complexity is exacerbated by the electrochemical reactions of the counterions at the metallic surface, but it will not be discussed in detail here.

In our model (see Fig. 1), a given volume of a liquid of constant viscosity $\mu$, surface tension $\gamma$, electrical conductivity $K$, and dielectric constant $\epsilon$ lies on a metallic plate held in a vacuum. A uniform electric field $E_{\infty}$ exists far from the liquid due to a high voltage applied between the plate an another distant parallel electrode. The wetted region of the plate is a circle of radius $a$, a condition that can be realized if the meniscus is attached to the edge of an orifice or crevice of radius $a$ drilled in the plate. A plate, rather than the needle or capillary used in a real ILIS to intensify the electric field, is assumed here merely to simplify the description of the far electric field.

The motion of the liquid in the meniscus is driven by the surface tension and electric stresses acting on its surface. The components of the electric stress normal and tangent to the surface are given by Eqs. (9a) and (9b) below. The inertia of the liquid and the mass flux across its surface are neglected, so that the flow obeys the Stokes equations, which express the balance of pressure and viscous forces, and the surface of the liquid is a material surface.

The electric fields in the liquid and in the vacuum are of the form $\boldsymbol{E}^{l}=-\boldsymbol{\nabla} \varphi^{l}$ and $\boldsymbol{E}=-\boldsymbol{\nabla} \varphi$, where $\varphi^{l}$ and $\varphi$ are the electric potentials. Since the effect of the space charge can be neglected and there is no free charge in the bulk of the liquid, these potentials satisfy Laplace's equation (in the liquid this result follows from the charge conservation condition 
$\boldsymbol{\nabla} \cdot \boldsymbol{j}=0$ with the current density given by Ohm's law $\boldsymbol{j}=\boldsymbol{K} \boldsymbol{E}^{l}$ with constant $K$ ). Boundary conditions for the electric potentials are $\varphi^{l}=\varphi=0$ at the metallic plate, the condition that the electric field tends to $E_{\infty}$ far from the plate, and the electrostatic conditions $\varphi^{l}=\varphi$ and $\epsilon_{0}\left(E_{n}-\epsilon E_{n}^{l}\right)=\sigma$ at the surface of the liquid Here, as in Sec. II B, the subscript $n$ denotes the component of the field normal to the surface and $\sigma$ is the density of free surface charge.

The density of free surface charge satisfies the transport equation $D \sigma / D t=\sigma \boldsymbol{n} \cdot \nabla \boldsymbol{v} \cdot \boldsymbol{n}+K E_{n}^{l}-j_{e}$ at the surface, which expresses that the surface charge is convected by the flow and the rate of change of the charge of a material element of the surface is equal to the rate at which conduction brings charge to the surface minus the rate at which the charge evaporates. Here $D \sigma / D t=\partial \sigma / \partial t+\boldsymbol{v} \cdot \nabla \sigma$ is the material derivative at the surface, $\boldsymbol{v}$ is the velocity of the liquid, $\boldsymbol{n}$ is the unit normal to the surface pointing away from the liquid, and the first term on the right-hand side of the transport equation is the effect of the surface strain.

In what follows distances are scaled with the radius of the contact line $a$, velocities with the viscous-capillary velocity $v_{c}=\gamma / \mu$, and electric fields with $E_{c}=\left(\gamma / \epsilon_{0} a\right)^{1 / 2}$, so that $\mu v_{c} / a, E_{c} a$, and $\epsilon_{0} E_{c}$ are used as the scales of pressure, electric potential, and surface charge density, respectively. The surface of the meniscus, which must be determined as part of the solution, is denoted by $f(\boldsymbol{x}, t)=0$, with $f(\boldsymbol{x}, t)<0$ in the liquid. Here $x=(x, r)$, where $x$ and $r$ are the distances to the plate and to the symmetry axis scaled with $a$, and $t$ is time scaled with $a / v_{c}$.

The governing equations and boundary conditions take the following dimensionless form:

$$
\begin{gathered}
\boldsymbol{\nabla} \cdot \boldsymbol{v}=0, \\
0=-\nabla p+\nabla^{2} \boldsymbol{v}, \\
\nabla^{2} \varphi^{l}=0
\end{gathered}
$$

in the liquid, for $f(x, t)<0$;

$$
\nabla^{2} \varphi=0
$$

in the vacuum, for $f(\boldsymbol{x}, t)>0$;

$$
\begin{gathered}
\boldsymbol{n} \cdot \tau \cdot \boldsymbol{n}+\boldsymbol{\nabla} \cdot \boldsymbol{n}=\tau_{n}^{e}, \\
\boldsymbol{t} \cdot \boldsymbol{\tau} \cdot \boldsymbol{n}=\tau_{t}^{e}, \\
\frac{D f}{D t}=0, \\
E_{n}-\epsilon E_{n}^{l}=\sigma, \\
\varphi=\varphi^{l}, \\
\frac{D \sigma}{D t}=\sigma \boldsymbol{n} \cdot \boldsymbol{\nabla v} \cdot \boldsymbol{n}+\Lambda E_{n}^{l}-D \sigma \exp \left(\beta E_{n}^{1 / 2}\right)
\end{gathered}
$$

at the surface $f(\boldsymbol{x}, t)=0$;

$$
\begin{gathered}
\boldsymbol{v}=0, \\
\varphi=\varphi^{l}=0
\end{gathered}
$$

at the plate, $x=0$; and

$$
\nabla \varphi=-B i
$$

far from the plate.

Here $\boldsymbol{n}$ and $\boldsymbol{t}$ are unit vectors normal and tangent to the surface of the liquid; $\tau=-p I+\tau^{\prime}$, where $p$ is the dimensionless pressure of the liquid, $I$ is the unit tensor, and $\tau^{\prime}$ is the dimensionless viscous stress tensor; $\tau_{n}^{e}$ and $\tau_{t}^{e}$ are the dimensionless components of the electric stress normal and tangent to the surface, given by

$$
\begin{gathered}
\tau_{n}^{e}=\frac{1}{2}\left(E_{n}^{2}-\epsilon E_{n}^{l^{2}}\right)+\frac{1}{2}(\epsilon-1) E_{t}^{2}, \\
\tau_{t}^{e}=\sigma E_{t},
\end{gathered}
$$

where $E_{n}=\boldsymbol{E} \cdot \boldsymbol{n}, E_{t}=\boldsymbol{E} \cdot \boldsymbol{t}$, and similarly for $\boldsymbol{E}^{\boldsymbol{l}}$, and $x$ and $\boldsymbol{i}$ are the distance to the solid plate and a unit vector normal to the plate. The solution of the problem depends on the six dimensionless parameters

$$
\begin{gathered}
\Lambda=\frac{\mu a K}{\epsilon_{0} \gamma}, \quad \epsilon, \quad B=\frac{E_{\infty}}{E_{c}}, \quad \mathcal{V}=\frac{V}{a^{3}}, \\
D=\frac{\mu a k T}{\gamma h} \exp \left(-\frac{\Delta G}{k T}\right), \quad \beta=\frac{1}{k T}\left(\frac{e^{3} E_{c}}{4 \pi \epsilon_{0}}\right)^{1 / 2},
\end{gathered}
$$

where $V$ is the volume of the meniscus, which is to be given as an initial condition and is conserved in its time evolution.

A stationary solution is found to exist only when $B$ is of order unity (see Sec. III A below). The dimensionless volume $\mathcal{V}$ is also taken to be of order unity, whereas $\epsilon$ is large for the liquids of interest. The values of the dimensionless conductivity $\Lambda$ and of $\beta$ depend on $a$. For a liquid with the typical values of the physical properties used in the estimates of Sec. II B, it is $\Lambda \sim 10^{6}$ and $\beta \sim 4$ when $a=100 \mu \mathrm{m}$, which is typical of a meniscus at the end of a capillary, and $\Lambda$ $\sim 100$ and $\beta \sim 40$ when $a=10 \mathrm{~nm}$, which might be used to simulate the evaporation region of the surface alone. Finally, real values of $D$ are very small and such that the last two terms of (6f) are of the same order in the evaporation region (cf. the estimates of Sec. II B). Here, to make the numerical problem affordable, the values of $\beta$ used in the computations are somewhat smaller than for a real source, and $D$ is increased accordingly to keep the order of the ion evaporation term in (6f) unchanged.

Axisymmetric solutions of the problem (4)-(9) have been computed using standard boundary element methods to solve the Laplace and Stokes equations (4a)-(4c) and (5) and a second-order Runge-Kutta method to advance the evolution equations (6c) and (6f). 

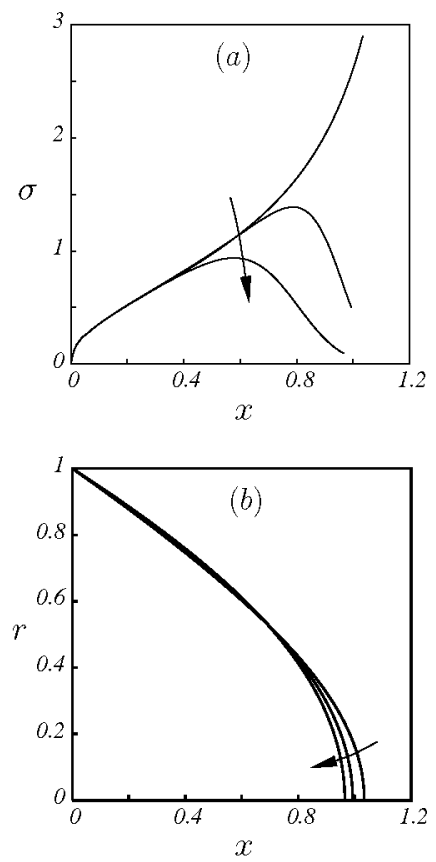

FIG. 2. Distributions of free surface charge (a) and shapes of the meniscus (b) for $\Lambda=10000, \epsilon=50, B=0.65, \mathcal{V}=1.5, \beta=10$, and the three values $D=0,10^{-4}$, and $10^{-3}$, increasing as indicated by the arrows.

\section{RESULTS AND DISCUSSION}

\section{A. Constant-volume menisci}

Stationary solutions of (4)-(9) for very polar liquids $(\epsilon \gg 1)$ are nearly hydrostatic irrespective of the intensity of ion evaporation, determined by $D$ and the dimensionless strength of the applied field $B$. This is because condition (6d) implies that $E_{n}^{l} \ll E_{n}$ for any $\sigma$ between zero and its equilibrium value $\sigma_{\mathrm{eq}}=E_{n}$. Since the solution of Laplace's Eq. (4c) implies that $E_{t} \sim E_{n}^{l}$, the electric field does not enter the liquid, and the electric shear (9b) is small compared with the normal electric stress (9a), which can be approximated by $\tau_{n}^{e}=\frac{1}{2} E_{n}^{2}$. This normal stress can be balanced by pressure and surface tension stresses, leaving the liquid at rest in first approximation. Hydrostatic solutions with $\sigma=\sigma_{\text {eq }}\left(\boldsymbol{E}^{l}=0\right)$ and $\sigma=0$ have been computed by Taylor Miksis and Wohlhuter and Basaran among others; see references in the last paper. Ion evaporation reduces the density of surface charge below its equilibrium value, making $0<\sigma<\sigma_{\text {eq }}$, but this induces only a weak motion in the liquid when $\epsilon$ is large.

Figure 2 illustrates this result. The density of surface charge around the tip of the meniscus decreases when $D$ increases, and ion evaporation approaches the conductioncontrolled regime discussed in Sec. II B for the largest value of $D$ shown. The shape of the meniscus, however, changes little with $D$ and nearly coincides with the hydrostatic solution (for $D=0$ ).

The length of the meniscus is shown in Fig. 3 as a function of the applied field for different values of the dimensionless volume of liquid. In line with the hydrostatic solutions the elongation of the meniscus increases with

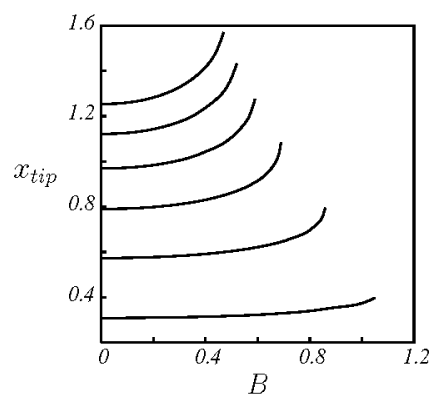

FIG. 3. Length of the meniscus as a function of the applied electric field for $\Lambda=10000, \epsilon=50, D=10^{-3}, \beta=10$, and $\mathcal{V}=0.5,1$, $1.5,2,2.5$, and 3 , increasing from bottom to top.

the applied field and reaches a maximum for a certain value of the field above which no stationary solution exists. The maximum value of the applied field decreases when the volume of liquid increases. The shape of the meniscus for this maximum field is given in Fig. 4 for various volumes of liquid. As can be seen, the meniscus stays smooth at its maximum elongation. The electric field at the surface and the rate of ion evaporation (not displayed) reach their maxima at the tip of the meniscus, but the region of the surface where the electric field is of the order of its maximum value is never small compared with the size of the meniscus. For small volumes of liquid, the meridional section of the meniscus becomes convex toward the liquid in a region around the contact line when the applied field is increased. The angle of the surface to the plate becomes zero for a certain value of the field, above which the surface of the liquid extends to the region $x<0$. These solutions are meaningless for a meniscus attached to a smooth solid surface (though they can be realized at the end of a capillary) and have not been computed. The lowest curve in Fig. 3, for $\mathcal{V}=0.5$, ends at the value of $B$ for which the surface of the liquid becomes tangent to the plate. The innermost curve in Fig. 4 is a meridional section of this surface.

These results may have a bearing on the observed differences between the emissions of capillary and externally wetted needle types of sources. In the former type of source the radius of the capillary (analogous to $a$ ) tends to be very large compared to the size of the evaporation region estimated in Sec. II B, which according to these results prevents the capillary sources from operating in the purely ionic regime. The sources may still work in the mixed (ions plus drops) regime because the high flow rate accompanying the emission of

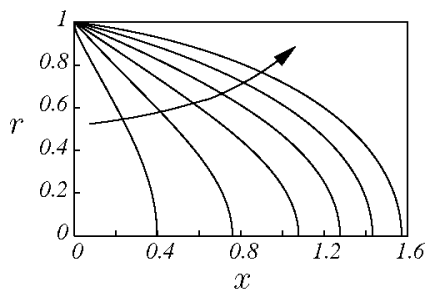

FIG. 4. Limiting shape of the meniscus for $\Lambda=10000, \epsilon=50$, $D=10^{-3}, \beta=10$, and $\mathcal{V}=0.5,1,1.5,2,2.5$, and 3 , increasing as indicated by the arrow. 
drops allows the meniscus to become a cone-jet confining the region of high surface field and ion evaporation to a close vicinity of the apparent apex of the cone.

If the characteristic size $r_{v}$ of the ion evaporation region is in the range of tens of nanometers, as in the estimates of Sec. II $\mathrm{B}$, then the result that in the purely ionic regime the minimum radius of curvature of the surface cannot be much smaller than the size of the meniscus also poses a strong limitation for externally wetted needle sources to work in this regime. The limitation becomes less strong if the solvation energy is smaller than assumed in Sec. II B (a possibility already mentioned in connection with the estimate of the electric current), because then the electric field needed at the surface to have ion evaporation is smaller than in Sec. II B and $r_{v}$ is larger. A conceivable possibility for very dilute solutions of ionic liquids in very volatile solvents, for which $v_{v}$ may be much larger than estimated in Sec. II B, is that the flow induced by ion and solvent evaporation might affect the shape of the meniscus and lead to a stationary but not hydrostatic tip somewhat sharper than in the computations of this section. This case is not covered by the present analysis. The effect of the evaporation-induced flow on the shape and stability of the meniscus has been discussed in the literature for LMISs.

The hydrostatic results of Wohlhuter and Basaran strongly suggest that there is a second branch of stationary solutions above the turning point shown in Fig. 3 for various volumes of liquid. At the high values of $\epsilon$ of interest here, this second branch should end in a pointed meniscus shortly beyond the turning point. Solutions of the second branch are unstable, if they exist, and therefore cannot be computed with the time-marching numerical method used here or realized in a stationary source.

The electric field and the density of surface charge at the tip of the meniscus are shown in Fig. 5 as functions of the applied field $B$. The electric field at the tip, which is the maximum field on the surface of the liquid, increases with $B$ and with the volume of liquid at constant $B$. The density of surface charge follows the same trends for moderate values of $B$, but it reaches a maximum at a certain $B$ and decreases when $B$ is further increased. The value of $B$ at which the maximum is attained decreases when the volume of liquid increases, but the maximum value of $\sigma$ at the tip does not depend on the volume. It decreases when $D$ in $(6 \mathrm{f})$ increases [see Fig. 2(a)] and increases with the dimensionless conductivity $\Lambda$. The density of surface charge may become very small before the meniscus reaches its maximum elongation [curves for $\mathcal{V}=1$ and 1.5 in Fig. 5(b)], which is the mark of conduction-controlled ion evaporation. This regime is not attained for small volumes [e.g., the curve for $\mathcal{V}=0.5$ in Fig. 5 (b)] because the surface of the meniscus becomes tangent to the plate before the applied field can be increased to sufficiently high values.

\section{B. Ion evaporation current}

Figure 6 shows the dimensionless ion evaporation current, which is the integral of $j_{e}$ over the surface of the meniscus scaled with $\epsilon_{0} E_{c} \gamma a / \mu$. The current is very small for moder-
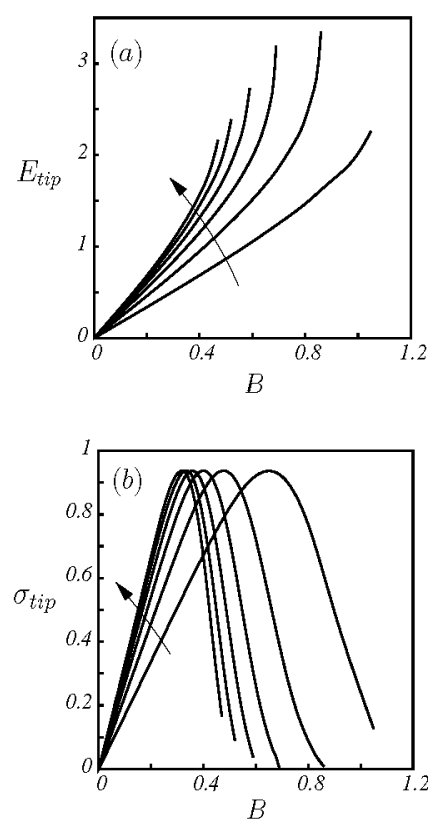

FIG. 5. Electric field (a) and density of free surface charge (b) at the tip of the meniscus as functions of the applied field for $\Lambda$ $=10000, \epsilon=50, D=10^{-3}, \beta=10$, and $\mathcal{V}=0.5,1,1.5,2,2.5$, and 3, increasing as indicated by the arrows.

ate values of the applied field $B$ and increases nearly linearly with $B$ in a region of larger values of this parameter, in which the current also increases with the electrical conductivity of the liquid ( $\Lambda$ in dimensionless variables). The transition between the two regions is already sharp for the value $\beta=10$ used in the computations, and becomes sharper when $\beta$ increases, though the numerical problem becomes then stiffer. This transition is consistent with the notion of a starting voltage found in experiments with both LMISs and ILISs

but it is not connected here with the formation of a conical meniscus. A conical meniscus and emission of drops will probably appear when $B$ increases above its value at the end point of each curve in Fig. 6, which mark the upper boundary of the purely ionic regime. A nearly linear currentvoltage characteristic in a bounded range of voltages (here of $B$ ) is found in experiments with ILISs and will be discussed below. It also occurs for LMISs, where it has been

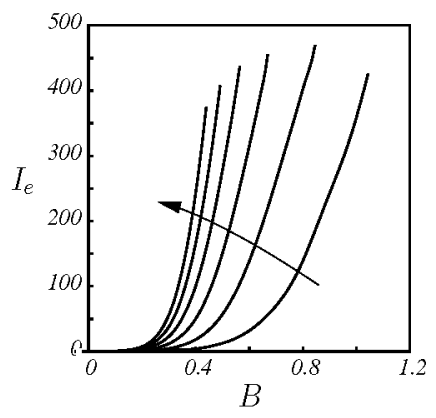

FIG. 6. Electric current as a function of the applied electric field for $\Lambda=10000, \epsilon=50, D=10^{-3}, \beta=10$, and $\mathcal{V}=0.5,1,1.5,2,2.5$, and 3 , increasing as indicated by the arrow. 
explained taking into account the effect of the space charge

The mass flux accompanying ion evaporation, whose effect has been left out of the formulation, is proportional to the electric current. If the mass flux could be directly controlled, as in a regular electrospray then the results of Fig. 6 would predict that the volume of the meniscus increases with the mass flux at constant voltage (constant $B$ ) and decreases when the voltage is increased at constant mass flux, which are well-known trends for the meniscus of an electrospray.

The results of Fig. 6 reflect the high sensitivity of the evaporation rate to the electric field. Ion evaporation is negligible in the nearly frozen regime of low $B$ and becomes independent of the kinetics (of $D$ and $\beta$ ) on most of the evaporating surface in the conduction-controlled regime of high $B$. A crossover electric field may be defined for which the last two terms of $(6 \mathrm{f})$, representing conduction of electric current from the liquid to the surface and ion evaporation, are equal to each other with $\epsilon E_{n}^{l}=\sigma$, so that the surface charge and the electric displacement in the liquid are equally important in (6d). The crossover field is

$$
E_{0}=\left(\frac{1}{\beta} \ln \frac{\Lambda}{\epsilon D}\right)^{2}
$$

When $\beta \gg 1$, the ion evaporation term is exponentially small in the region of the surface where $E_{n}<E_{0}$, and the density of surface charge is exponentially small in the region where $E_{n}>E_{0}$. In addition $E_{n}^{l} \approx 0\left(\sigma \approx E_{n}\right)$ in the former region, because the motion of the liquid is very weak in a stationary meniscus and conduction has plenty of time to accumulate electric charge at the surface and screen the liquid from the outer field. The stationary solution of (4)-(9) has therefore a simple asymptotic structure for large values of $\epsilon$ and $\beta$. The liquid is at rest and its surface is an equipotential of the outer electric field $(\varphi=0)$. The shape of the meniscus is independent of ion evaporation. The electric field in the liquid is small, of $O(1 / \epsilon)$, and is given by the solution of $(4 \mathrm{c})$ with the conditions $E_{n}^{l}=0$ in the region of the surface where $E_{n}<E_{0}$ and $E_{n}^{l}=E_{n} / \epsilon$ in the region where $E_{n}$ $>E_{0}$. The ion evaporation current coincides with the current reaching the latter region of the surface by conduction in the liquid.

The distributions of density of surface charge and of density of evaporation current normalized with its value at the tip are shown in Fig. 7 for $\epsilon=50, \beta=10$, and various values of the applied field $B$. The maximum density of surface charge is nearly independent of the applied field and is not far from half of the value of the crossover field $\left(E_{0} \approx 1.49\right.$ for the values of the parameters used in these computations), which is in agreement with the qualitative asymptotic description; see also Fig. 5(b) above. The normalized distributions of density of evaporation current depend little on $B$ because the shape of the meniscus changes little with this parameter. This, together with the nearly linear variation of the field at the tip with $B$ linear variation of the current in Fig. 6.
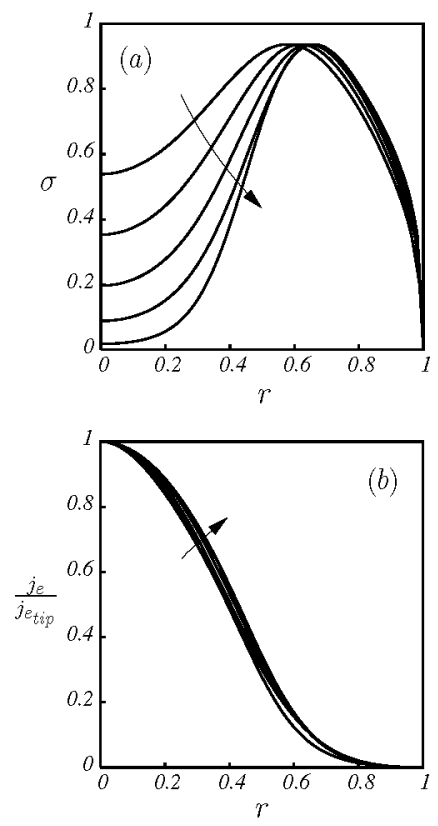

FIG. 7. Distributions of free surface charge (a) and normalized evaporation current density $(b)$ on the surface of the meniscus for $\Lambda=10000, \epsilon=50, \mathcal{V}=1, D=10^{-3}, \beta=10$, and $B=0.65,0.70,0.75$, 0.80 , and 0.85 , increasing as indicated by the arrow.

\section{Force and feeding flow}

The force exerted by the liquid on the metallic plate, scaled with $\gamma a$, is

$$
F=2 \pi \int_{0}^{1}\left(\boldsymbol{i} \cdot \tau \cdot \boldsymbol{i}+\frac{1}{2} \epsilon E^{l^{2}}\right) r d r
$$

where the first term includes the pressure and viscous stresses and the second term is the Maxwell electric stress, which is small when $\epsilon$ is large. This force is shown in Fig. 8 (solid contours) as a function of the applied field and the volume of the meniscus, $B$ and $\mathcal{V}$. As was seen before, stationary solutions exist only in a certain region of the $B-\mathcal{V}$ plane. For a given $\mathcal{V}$, the range of possible $B$ extends from

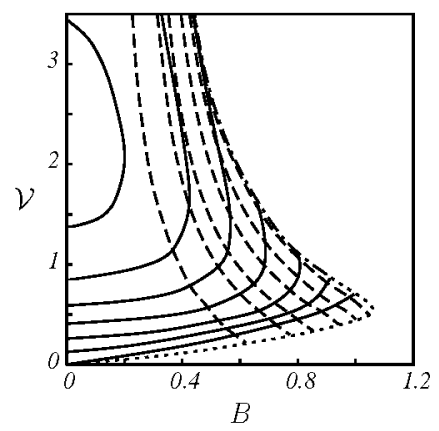

FIG. 8. Isolines of the force on the plate (12) (solid) and the electric current $I_{e}$ (dashed) as functions of $B$ and $V$ for $\Lambda=10000$, $\epsilon=50, D=10^{-3}$, and $\beta=10$. Contours displayed are $F=0,-1,-2$, $-3,-4,-5$, and -6 , decreasing from right to left, and $I_{e}=10,50$, $100,200,300$, and 400 , increasing from left to right. 
zero to the value at the turning point in Fig. 3 (upper boundary, dash-dotted line, in Fig. 8) or to the value for which the surface of the liquid becomes tangent to the plate (lower boundary, dotted line, in Fig. 8). The force $F$ is negative (toward the plate) in most of this region, and one solution or two solutions with different volumes exist for given values of $F$ and $B$. The dashed curves in Fig. 8 are contours of constant electric current, which increases on approaching the right-hand side boundary of the region of existence of stationary solutions.

Solutions of (4)-(9) have been computed up to this point for given values of the volume of liquid, but this volume is not known from the outset in an ILIS. A more realistic condition, though still short of a detailed analysis of the feeding flow in the capillary or around the needle of an ILIS, can be obtained modeling this flow by a pressure drop proportional to the flow rate entering the meniscus

$$
p_{0}+F=\alpha Q
$$

where $Q$ is the flow rate, $p_{0}$ is the pressure of the liquid in a reservoir upstream of the meniscus, the mean pressure at the base of the meniscus is taken as $-F$ computed from (12), and $\alpha$ is a proportionality constant.

Estimates for a drag-free (roughened needle surface) LMIS suggest that the capillary depression in the grooves of the needle could deliver a flow rate to the meniscus much higher than that needed for ion evaporation In these conditions, the latter flow rate can be omitted from the righthand side of (13), leaving only the flow rate required to change the volume of the meniscus in a transient, $Q$ $=\mathrm{d} \mathcal{V} / \mathrm{d} t$. This flow rate is zero for a stationary solution, for which (13) reduces to the constant-pressure condition

$$
F=-p_{0}
$$

Transient solutions have been computed by replacing the constant-volume condition (7a) by (13) with $Q=\mathrm{d} \mathcal{V} / \mathrm{d} t$; i.e., setting $\boldsymbol{v}=\alpha^{-1}\left(p_{0}+F\right) v_{0}(r) \boldsymbol{i}$ at the plate instead of (7a). Here $v_{0}=0$ for $r>1$ and $2 \pi \int_{0}^{1} v_{0} r d r=1$, while results obtained for different functions $v_{0}(r)$ that satisfy these conditions are very similar. Transient solutions for given values of $B$ and $p_{0}$, with $\alpha=O(1)$, tend to the lower stationary solution of Fig. 8 with $F=-p_{0}$. The lower branch of Fig. 8 is therefore stable and the upper branch is unstable when it exists. This result is an extension to electrified menisci of a well-known result for attached drops subject to surface tension forces only. It may be worth noting, however, that some solutions of the upper branch seem to be stabilized, and some solutions of the lower branch destabilized, when (13) is replaced by (14); i.e., when $F=-p_{0}$ during the transient, which is the limiting form of (13) for $\alpha \rightarrow 0$.

The electric current and the volume of the meniscus for stationary solutions with $p_{0}=2$ and $p_{0}=5$ are shown as functions of the applied electric field by the solid curves in Fig. 9. These solutions lie on the lower branch of the contours $F$ $=-p_{0}$ in Fig. 8 . The electric current can only reach moderate values when $p_{0}=5$ because the applied field cannot increase beyond the turning point in this figure. The region of high
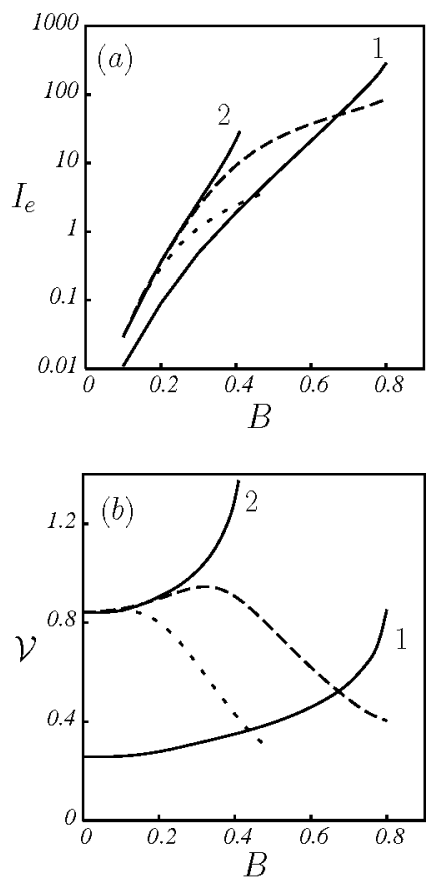

FIG. 9. Electric current (a) and volume of the meniscus (b) as functions of the applied electric field for $\left(p_{0}, \hat{\alpha}\right)=(2,0)$ (solid curve 1), $(5,0)$ (solid curve 2), (5,0.06) (dashed curve), and $(5,1)$ (dotted curve). Values of other parameters are $\Lambda=10000, \epsilon=50, D=10^{-3}$, and $\beta=10$.

current on the right-hand side in Fig. 8 is accessible only to solutions with low values of the feeding pressure $p_{0}$, which have small menisci.

This situation may change when the pressure drop induced in the feeding line by the flow rate that accompanies evaporation is taken into account, as was done by Mair

for a LMIS and by Lozano and Martínez-Sánchez in their discussion of the flow around the externally wetted needle of an ILIS. The direct effect of this flow rate on the meniscus has been left out of the formulation of Sec. II C, but the value of the flow rate can be easily computed, being proportional to the evaporation current $I_{e}$, and included on the right hand side of (13). The boundary condition at the plate is then modified to $\boldsymbol{v}=\alpha^{-1}\left(p_{0}+F-\hat{\alpha} I_{e}\right) v_{0}(r) \boldsymbol{i}$, so that $F-\hat{\alpha} I_{e}=-p_{0}$ in the final stationary state. Here $\hat{\alpha}$ is a dimensionless measure of the hydraulic resistance of the needle, whose value should be determined by an analysis of the flow feeding the meniscus. Two sample solutions for $p_{0}=5$ and different values of $\hat{\alpha}$ are given by the dashed and dotted curves in Fig. 9. The decrease of the slope of the $I_{e}-B$ characteristic displayed by these curves with increasing $\hat{\alpha}$ is consistent with observed differences between the characteristics of drag-free LMISs and sources with non-roughened emitters

The path of the solution in the $B-\mathcal{V}$ plane in Fig. 8 depends on $\hat{\alpha}$. Appropriate combinations of values of this parameter and $p_{0}$ allow the path to approach the region of high electric current. These results confirm the important effect of the mechanism used to feed the meniscus on the characteristic of an ILIS. 


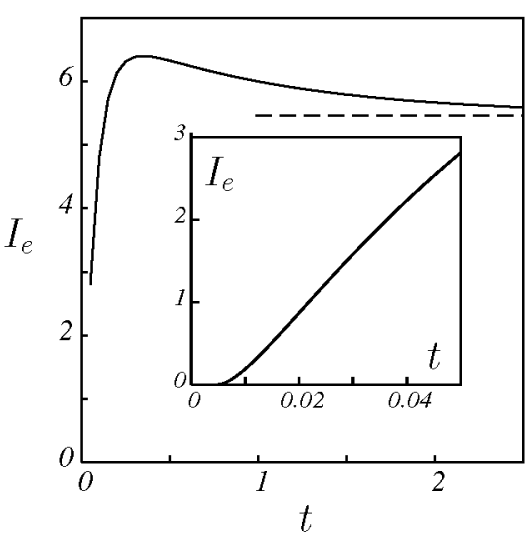

FIG. 10. Electric current as a function of time (scaled with $a \mu / \gamma$ ) during the transient that follows a step change of the applied electric field from $B=-0.8$ to 0.6 at $t=0$. Values of other parameters are $\Lambda=500, \epsilon=50, \mathcal{V}=1, D=5 \times 10^{-5}$, and $\beta=10$.

\section{Dynamic response to voltage alternation}

Low-frequency alternation of the voltage between positive and negative values is required in an ILIS to avoid the electrochemical decomposition of the liquid at the surface of the emitter This makes the flow and the emitted current time periodic, but the stationary solutions discussed above are still applicable during most of the period because the response time of the source to a change of the voltage is short compared with the time between successive changes. Focusing on this transient response, Lozano and MartínezSánchez observed a delay of the ion current onset relative to the nearly square-shaped (switch time of about $200 \mu \mathrm{s}$ ) voltage signal applied to their sources, with a characteristic current overshoot often following the current delay

They measured the delay as a function of the positive and negative voltages and the alternation frequency, and qualitatively explained their results in terms of the flow induced by surface tension and electric stresses in the grooves on the roughened surface of the needle and in the smooth convex surface around its tip.

As a preliminary attempt to examine the role played by the meniscus in this transient response, Eqs. (4)-(9) have been solved for a given value of $B$ using as initial condition the stationary solution for a different $B$ of opposite sign. The other parameters in (10) are left constant in these computations, though $\mathcal{V}$ and $D$ could also take different values in each of the two parts of the voltage alternation cycle. The factor $\sigma$ in the last term of $(6 f)$ is changed to $\max (\sigma B, 0) / B$ because ion evaporation should occur only when the surface charge and the electric field have the same sign.

Figure 10 shows the time evolution of the current for a sample computation in which $B$ is abruptly changed from -0.8 to 0.6 at $t=0$. For a typical solution of an ionic liquid in propylene carbonate, the value $\Lambda=500$ used in this computation corresponds to a tiny meniscus with $a=0.05 \mu \mathrm{m}$. The numerical results display a short delay and a small overshoot of the current, but these features cannot correspond to the experimental results The delay (visible in the inset of the figure) is due to the time required by conduction to bring positive charge to the surface and evacuate the negative charge that was initially there. The characteristic time of this process is the electric relaxation time of the liquid $t_{e}$ $=\epsilon_{0} \epsilon / K(\epsilon / \Lambda$ in dimensionless variables $)$ or a time even shorter than this in the conduction-controlled evaporation regime, in which the dimensionless $\sigma$ is very small. The time $t_{e}$ is far too short compared with the voltage switch time scale in the experiments of Lozano and Martínez-Sánchez

for charge relaxation effects to account for the delay reported by these authors.

The overshoot reflects the existence of another process with a different characteristic time. The stationary meniscus for $B=0.6$ is more rounded than the initial meniscus for $B$ $=-0.8$, but the characteristic time of adjustment of the surface is the viscous capillary time $t_{c}=a / v_{c}$ (equal to unity in dimensionless variables), which is large compared with the electric relaxation time. In these conditions, conduction brings positive charge and ion evaporation sets in at a surface that is more elongated, and therefore causes a stronger intensification of the field, than the final stationary surface. The field at the surface and the electric current take therefore large values that slowly decrease as the surface recedes in a dimensionless time of $O(1)$. For the probable sizes of the menisci the viscous capillary time is only marginally shorter than the voltage switch time, but the overshoot in Fig. 10 becomes an undershoot

when the initial shape of the meniscus is more rounded than its final shape.

These negative results strongly suggest that the flow in the grooves and the smooth surface of the needle, rather than the meniscus, causes the observed response of the sources to voltage alternation. This flow introduces larger characteristic times in the dynamics scope of the model (4)-(9).

The numerical results of this section also illustrate a certain robustness of the meniscus. Although the numerical computations have not been exhaustive, the meniscus was never observed to disrupt or shed charged drops during the transient, contrary to what could be expected for a cone-jet subjected to similar voltage alternation.

\section{CONCLUSIONS}

A qualitative model of the purely ionic regime of an ILIS has been formulated. An order-of-magnitude analysis of the flow and the electric field in the meniscus of the source suggests that the effects of the space charge, the mass flow accompanying ion evaporation, and the inertia of the liquid are small in this regime and can be neglected in first approximation. The ion evaporation current is limited by the finite conductivity of the liquid rather than by the space charge around its surface, in contrast to the case of a LMIS.

Using these results, the flow in the meniscus and the ion evaporation current can be largely isolated from the complexities of the flow around the needle or in the capillary of the source. Stationary menisci of very polar liquids are found to be nearly hydrostatic and to exist only for values of the applied field below a certain maximum, at which the meniscus attains its maximum possible elongation but stays 
smooth. Owing to the high sensitivity of the ion evaporation rate with the electric field, the current/applied field characteristic displays a region of very weak ion evaporation for values of the applied field below a certain starting field, and a conduction-controlled region for higher values of the applied field. A qualitative description is given of the asymptotic structure of the solution in the latter region.

The model of the meniscus, supplemented with a simplified treatment of the flow around the needle or in the capillary as an effective feeding line, predicts that, for drag-free sources (no pressure drop in the effective feeding line), the size of the meniscus decreases and the maximum attainable current increases when the feeding pressure is decreased, though the voltage required to extract this current increases. Appropriate combinations of feeding pressure and pressure drop may lead to high maximum currents.

\section{ACKNOWLEDGMENTS}

This work was supported by the Spanish Ministerio de Educación y Ciencia through Projects Nos. DPI2004-05246C04-02 and DPI2007-66659-C03-02.
P. D. Prewett and G. L. R. Mair, Focused Ion Beams from LMIS (Wiley, New York, 1991).

C. Bartoli, H. von Rohden, S. P. Thompson, and J. Blommers, J. Appl. Phys. 17, 2473 (1984).

R. G. Forbest, Vacuum 48, 85 (1997).

J. Fernández de la Mora, Annu. Rev. Fluid Mech. 39, 217 (2007).

J. Fernández de la Mora and I. G. Loscertales, J. Fluid Mech. 260, 155 (1994).

J. B. Fenn, M. Mann, C. K. Meng, S. K. Wong, and C. Whitehouse, Science 246, 64 (1989).

R. J. Pfeifer and C. D. Hendricks, AIAA J. 6, 496 (1968).

M. N. Huberman and S. G. Rosen, J. Spacecr. Rockets 11, 475 (1974).

M. Martínez-Sánchez, J. Fernández de la Mora, V. Hruby, M. Gamero-Castaño, and V. Khayms, in Research on Colloid Thrusters, Proceedings of the 26th IEPC, Electric Rocket Propulsion Society, Kitakyushu, Japan, 1999 (unpublished), pp. 93-100.

M. Gamero-Castaño and V. Hruby, J. Propul. Power 17, 977 (2001).

M. Gamero-Castaño and J. Fernández de la Mora, J. Mass Spectrom. 35, 790 (2000).

J. Perel, J. F. Mahoney, R. D. Moore, and A. Y. Yahiku, AIAA J. 7, 507 (1969).

I. Romero-Sanz, R. Bocanegra, J. Fernández de la Mora, and M. Gamero-Castaño, J. Appl. Phys. 94, 3599 (2003).

I. Romero-Sanz and J. Fernández de la Mora, J. Appl. Phys. 95, 2123 (2004).

P. Lozano and M. Martínez-Sánchez, J. Colloid Interface Sci. 282, 415 (2005).

P. Lozano, J. Phys. D 39, 126 (2006).

C. Larriba, S. Castro, J. Fernández de la Mora, and P. Lozano, J. Appl. Phys. 101, 084303 (2007).

S. Castro, C. Larriba, J. Fernández de la Mora, P. Lozano, S. Sümer, Y. Yoshida, and G. Saito, J. Appl. Phys. 102, 094310 (2007).

G. K. Van Berkel and V. Kertesz, Anal. Chem. 79, 2778 (2007).

J. O. Bockris and A. K. N. Reddy, Modern Electrochemistry I:
Ionics (Plenum, New York, 1998); J. O. Bockris, A. K. N. Reddy, and M. Gamboa-Aldeco, Modern Electrochemistry 2A: Fundamentals of Electrodics (Kluwer Academic/Plenum, Dordrecht, 2000).

P. Lozano and M. Martínez-Sánchez, J. Colloid Interface Sci. 280, 149 (2004).

P. Lozano and M. Martínez-Sánchez, J. Phys. D 38, 2371 (2005).

J. V. Iribarne and B. A. Thomson, J. Chem. Phys. 64, 2287 (1976).

I. G. Loscertales and J. Fernández de la Mora, J. Chem. Phys. 103, 5041 (1995).

M. Gamero-Castaño and J. Fernández de la Mora, J. Chem. Phys. 113, 815 (2000).

M. Gamero-Castaño and J. Fernández de la Mora, Anal. Chim. Acta 406, 67 (2000).

M. Labowsky, J. B. Fenn, and J. Fernández de la Mora, Anal. Chim. Acta 406, 105 (2000).

R. F. Probstein, Physicochemical Hydrodynamics. An Introduction (Butterworth, London, 1989), Chaps. 3 and 6.

I. Guerrero, R. Bocanegra, F. J. Higuera, and J. Fernández de la Mora, J. Fluid Mech. 591, 437 (2007).

L. D. Landau and E. M. Lifshitz, Electrodynamics of Continuous Media (Pergamon, Oxford, 1960).

D. A. Saville, Annu. Rev. Fluid Mech. 29, 27 (1997).

F. J. Higuera, Phys. Rev. E 69, 066301 (2004).

S. P. Thompson and P. D. Prewett, J. Phys. D 17, 2305 (1984).

G. K. Batchelor, An Introduction to Fluid Dynamics (Cambridge University Press, Cambridge, UK, 1967), p. 132.

G. I. Taylor, Proc. R. Soc. London, Ser. A 280, 383 (1964).

M. J. Miksis, Phys. Fluids 24, 1967 (1981).

F. K. Wohlhuter and O. A. Basaran, J. Fluid Mech. 235, 481 (1992).

A. Wagner, J. Vac. Sci. Technol. 16, 1871 (1979).

D. R. Kingham and L. W. Swanson, Appl. Phys. A: Solids Surf. 34, 123 (1984).

A. E. Bell and L. W. Swanson, Appl. Phys. A: Solids Surf. 41, 335 (1986).

G. L. R. Mair, J. Phys. D 30, 1945 (1997).

J. Fernández de la Mora (private communication). 